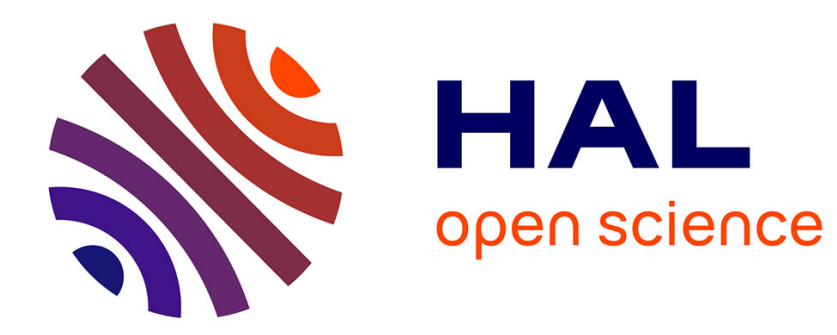

\title{
Représentations et médiation du patrimoine mondial : Le site d'Arequipa
}

\author{
Mathieu Dormaels
}

\section{To cite this version:}

Mathieu Dormaels. Représentations et médiation du patrimoine mondial : Le site d'Arequipa. Culture et Musées, 2021, 23, pp.109-138. 10.4000/culturemusees.1376 . hal-03265454

\section{HAL Id: hal-03265454 \\ https://hal.science/hal-03265454}

Submitted on 20 Jun 2021

HAL is a multi-disciplinary open access archive for the deposit and dissemination of scientific research documents, whether they are published or not. The documents may come from teaching and research institutions in France or abroad, or from public or private research centers.
L'archive ouverte pluridisciplinaire HAL, est destinée au dépôt et à la diffusion de documents scientifiques de niveau recherche, publiés ou non, émanant des établissements d'enseignement et de recherche français ou étrangers, des laboratoires publics ou privés. 


\section{(2) OpenEdition Journals}

\section{Culture \& Musées}

Muséologie et recherches sur la culture

$23 \mid 2014$

Tourisme et médiations des patrimoines

\section{Représentations et médiation du patrimoine mondial : Le site d'Arequipa}

Representation and Mediation of the World Heritage : The Historic Center of Arequipa

Representaciones y mediación del patrimonio mundial : El sitio de Arequipa

\section{Mathieu Dormaels}

p. $109-138$

https://doi.org/10.4000/culturemusees.1376

\section{Résumés}

Français English Español

Cet article s'interroge sur l'influence du tourisme dans les processus de patrimonialisation et de médiation liés à la mise en tourisme du centre historique d'Arequipa (Pérou), inscrit sur la Liste du patrimoine mondial. L'étude met en évidence des relations entre l'évolution des représentations, les transformations du site patrimonial et la médiation du patrimoine. Ces influences proviennent principalement de l'énoncé d'une valeur universelle exceptionnelle, qui justifie l'inscription au patrimoine mondial, mais aussi d'une mise en abyme des représentations par l'anticipation des attentes des touristes. Ce phénomène produit alors des représentations «méta-patrimoniales » dont le centre historique lui-même devient l'outil de médiation.

In this paper, we examine the influence of tourism in heritage building and mediation processes, related to the touristification process in the historic center of Arequipa (Peru), inscribed on the World Heritage List. This study highlights the relationship between the evolution of representations, site transformations and the mediation of heritage. These influences mainly come from the statement of outstanding universal values, which justifies the World Heritage inscription, but also from a mise en abyme of representations by anticipating the expectations of tourists. This produces representations that turn the historic center itself into the main tool of mediation.

En este artículo, nos preguntamos sobre la influencia del turismo en los procesos de patrimonilización y de mediación, vinculados a las prácticas turísticas en el centro histórico de Arequipa (Perú), inscrito sobre la Lista del patrimonio mundial. Esta investigación evidencia las relaciones entre la evolución de las representaciones, las transformaciones del sitio patrimonial y la mediación del patrimonio. Estas influencias provienen principalmente de la declaración de valor universal excepcional, que justifica la inscripción al patrimonio mundial, pero también de una mise en abyme de las representaciones con la anticipación de las expectativas de los turistas. Esto produce representaciones del patrimonio que transforman al mismo centro histórico en una herramienta de mediación.

\section{Entrées d'index}

Mots-clés : tourisme, patrimoine mondial, médiation, Arequipa, Pérou

Keywords: tourism, world heritage, mediation, Arequipa, Peru

Palabras clave: turismo, patrimonio mundial, mediación, Arequipa, Perú

Notes de la rédaction

Manuscrit reçu le : 6 juin 2013

Version révisée après expertise, reçue le $: 18$ décembre 2013

2e version révisée, reçue le : 23 février 2013

Article accepté pour publication le : 5 mars 2014

\section{Texte intégral}

Cet article est produit dans le cadre d'une recherche postdoctorale financée par une bourse du fonds québécois de recherche Société et Culture. L'auteur remercie les évaluateurs de ce texte ainsi qu'Émilie Flon pour leurs commentaires qui ont permis d'enrichir cet article.

1 Dans l'imaginaire touristique (Urbain, 1998 ; Amirou, 2000), les sites inscrits sur la Liste du patrimoine mondial occupent une place à part. Ces lieux représentent une altérité, une culture différente de celle du touriste, tout en étant 
sujets à une appropriation particulière, des communautés et des visiteurs, liée à leur valeur universelle.

2 En effet, ces sites sont tout autant des marqueurs des identités locales, relevant des autorités locales et nationales, que des repères pour l'ensemble de l'humanité. Mais plus encore, ils réunissent des pratiques touristiques distinctes dont certaines relèvent du tourisme de masse - circuits organisés, visites groupées, etc.- et d'autres d'un tourisme qualifié de « culturel ». Ce sont des sites complexes, tant pour leur gestion qui implique de nombreuses parties prenantes que dans les significations symboliques qu'ils portent, à la fois comme attractions touristiques et sites patrimoniaux. Il s'agit donc de sites « hybrides ", c'est-à-dire qu'ils résultent d'une construction sociale reposant sur plusieurs cultures - populaire, folklorique, occidentale, autochtone, etc. -, et dont l'analyse et la compréhension requièrent une approche multidisciplinaire.

3 C'est à partir de cette approche de construction sociale que nous avons étudié la patrimonialisation du site inscrit au patrimoine mondial, en 2000 : le centre historique de la ville d'Arequipa au Pérou. Dans un premier temps, nous avons notamment voulu mettre en évidence et comprendre les liens entre les transformations physiques de l'environnement urbain et l'appropriation locale par la construction de nouvelles représentation ssociales (Dormaels, 2013). Au-delà de ces liens, on peut s'interroger sur les raisons qui motivent ces transformations. Or, s'agissant d'un site inscrit recevant un nombre significatif de touristes, soit en moyenne 160 ooo par an dans les cinq dernières années (Mincetur, 2013, données compilées par l'auteur), on peut questionner l'influence du tourisme dans ce processus de patrimonialisation. Celle-ci pourrait notamment se manifester à travers les gestes volontaires de mise en tourisme du centre-ville historique, mais aussi par l'intégration de représentations sociales associées à ces sites, produites ou transformées par les professionnels du tourisme. En effet, si dans le contexte du patrimoine mondial le tourisme participait de la patrimonialisation des sites, voire la motivait, alors la question de la médiation du patrimoine pour les touristes se poserait tout particulièrement puisqu'ils en seraient les principaux destinataires.

$4 \quad$ En d'autres termes, en quoi les représentations sociales produites ou transformées par les professionnels du tourisme influencent-elles la production de significations patrimoniales et leurs évolutions ? Plus largement, notre objectif est de contribuer à une meilleure compréhension du rôle du phénomène touristique dans la construction des représentations qui constituent le caractère patrimonial des sites urbains inscrits au patrimoine mondial.

5 Pour répondre à cette question, dans le cas de la ville d'Arequipa, nous donnerons d'abord une brève définition du patrimoine, des représentations patrimoniales et de la médiation telle que nous l'entendons dans cet article. Ensuite, au travers d'une courte revue de la littérature, nous verrons de quelle manière le tourisme et le patrimoine peuvent être liés, et comment l'approche théorique de cette relation a évolué, passant du tourisme culturel au tourisme patrimonial. Cela nous permettra de comprendre la relation particulière qui existe aujourd'hui entre le tourisme et le patrimoine dans le cas des sites inscrits sur la Liste du patrimoine mondial de l'Unesco. Puis nous verrons comment, par l'analyse des données collectées, des différents documents et des produits et pratiques touristiques, nous avons pu mettre en évidence dans notre étude du cas d'Arequipa l'influence des représentations sociales produites par les professionnels du tourisme sur la patrimonialisation et la transformation des valeurs patrimoniales et du patrimoine lui-même. Enfin, nous conclurons en proposant une nouvelle notion permettant de mieux décrire les relations entre les processus de patrimonialisation et la mise en tourisme du patrimoine dans les sites du patrimoine mondial.

\section{Patrimoine et tourisme : deux constructions sociales}

$6 \quad$ Les liens entre patrimoine et tourisme ont été étudiés dans plusieurs disciplines, notamment en géographie, en études urbaines, en sciences de l'information et de la communication (Davallon, 1991 ; Lazarrotti, 2000 ; Robinson, 2000 ; Morisset, 2009 ; Gravari-Barbas et al., 2011), voire en études touristiques et en économie où la désignation patrimoniale, tout particulièrement celle de l'inscription sur la Liste du patrimoine mondial, est même parfois assimilée à un " label » touristique (Van der Aa, 2005 ; Marcotte \& Bourdeau, 2008). Le tourisme est parfois abordé comme une « mise en marché » du patrimoine, parfois comme un mode de gestion du patrimoine au service du développement local (Patin, 2005), le patrimoine y étant considéré comme un fait et non pas comme une construction sociale. D’autres études, menées plutôt selon une approche sociologique (Cousin \& Réau, 2009), envisagent le tourisme comme l'élaboration de représentations, en l'occurrence d'attractions touristiques, décrites et appelées « sacralisation » par Dean MacCannell (1999). Parallèlement, depuis une quinzaine d'années, de nombreuses études ont abordé le patrimoine dans une perspective transdisciplinaire, comme le processus d'attribution d'une signification, une construction sociale, appelé «patrimonialisation » (Drouin, 2005 ; Davallon, 2006), dont Lucie K. Morisset (2009) signale d'ailleurs la similarité avec la sacralisation de D. MacCannell. Cette auteure souligne non seulement la relation conceptuelle, mais aussi l'émergence concomitante du patrimoine et du tourisme au Québec dans les années vingt, alors qu'apparaissent des plaques énonçant les reconnaissances patrimoniales le long des routes de la «belle province » empruntées par les touristes venus des États-Unis.

7 Cette approche de la patrimonialisation permet le rapprochement avec le tourisme en tant qu'il s'agit de deux phénomènes sociaux reposant sur la mise en œuvre de représentations. Dans le cas du patrimoine, nous reprenons ici l'approche développée précédemment (Dormaels, 2013) sur la base des travaux de Jean Davallon (1992) qui définit un dispositif médiatique producteur de sens. Dans cette perspective, nous entendons par " représentations sociales » les significations symboliques produites par le dispositif, c'est-à-dire par les acteurs sociaux qui y sont impliqués, et sur lesquelles reposent les valeurs patrimoniales. Conséquemment, la médiation est comprise ici comme l'expression des représentations sociales, ou encore comme la manifestation sensible de significations symboliques à travers des outils de médiation intentionnels - plans, brochures, panneaux, guides, etc. - ou non intentionnels - presse, publicités, choix d'aménagement urbains, etc.

8 Dans le cas qui nous occupe ici, nous nous intéressons particulièrement aux représentations sociales produites par les professionnels du tourisme, ou celles générées par d'autres acteurs comme les institutions locales, nationales ou internationales, et qui sont reprises puis transformées par les professionnels du tourisme. Notons d'ailleurs que, dans le cadre de cette étude, l'ensemble de ces professionnels regroupe aussi bien ceux de l'industrie et des entreprises privées qui proposent des visites guidées, qui produisent de l'information (éditeurs de guides touristiques) ou fournissent des services connexes comme l'hôtellerie et la restauration - que ceux qui travaillent dans les institutions publiques au développement du tourisme ou à la gestion et à l'aménagement des espaces touristiques. Tous ces acteurs intègrent divers 
éléments patrimoniaux à leurs offres de produits et services, en justifiant leur mise en tourisme par l'intérêt que cela suscite pour le patrimoine et pour sa conservation. Pourtant, le tourisme a longtemps été perçu comme un ennemi de la conservation du patrimoine ou au mieux comme un mal nécessaire permettant de générer les ressources financières nécessaires à la restauration et à l'entretien des sites. Cependant, à l'instar d'Olivier Lazzarotti (2003 : 92), l'idée est maintenant largement admise que « le tourisme et le patrimoine sont deux phénomènes aujourd'hui conjoints ».

$9 \quad$ Selon les cas, les autorités locales cherchent à mettre en tourisme un patrimoine existant, ou alors c'est le tourisme qui porte un regard nouveau sur un élément patrimonial et en modifie la signification. Dans certains cas extrêmes, le tourisme semble néanmoins transformer « dangereusement » le patrimoine. On observe alors que l'intensité élevée des activités touristiques provoque des changements d'usages qui, par exemple dans le patrimoine urbain, peuvent conduire à la disparition totale ou partielle de la fonction résidentielle et vider un quartier de ses habitants - Le Mont-SaintMichel, Venise, etc. Dès lors, l'expérience touristique elle-même s'en trouve aussi changée. Dans tous les cas, l'évolution du tourisme vers une industrie basée sur des ressources plutôt que sur des services, et le déplacement du patrimoine, sinon dans le marché, au moins dans des dynamiques relevant d'une « économie culturelle " (Benhamou, 2012), font qu'il est bien difficile aujourd'hui d'aborder l'un sans l'autre.

\section{Le tourisme culturel et patrimonial}

Bien que l'industrie et les professionnels du tourisme utilisent surtout l'expression " tourisme culturel », l'étude académique de la notion met en évidence des évolutions qu'il convient de distinguer. En effet, depuis une quarantaine d'années, le tourisme culturel s'est transformé et l'émergence de la notion de patrimoine a contribué à certains de ces changements. Pour comprendre quels sont les liens entre tourisme et patrimoine aujourd'hui, il apparaît donc utile de retracer l'évolution de la notion de tourisme culturel et l'autonomisation progressive du tourisme patrimonial dans la sphère académique. Ceci permettra notamment de mieux comprendre la situation particulière du patrimoine mondial en tant que lieu de pratiques touristiques massives et diverses, et qui constitue un contexte particulier pour l'étude des influences des professionnels du tourisme sur les représentations sociales et la construction du patrimoine.

11 La notion de tourisme culturel peut sembler difficile à circonscrire car, comme l'ont souligné déjà plusieurs auteurs (Knafou, 2009 : 11-12 ; McKercher \& du Cros, $2002: 1$ ), la pratique du tourisme a d'abord été culturelle, que l'on fasse remonter son origine aux Romains, au "Grand Tour » des aristocrates anglais ou à l'apparition des premières destinations thermales. On pourrait d'ailleurs défendre l'idée que le tourisme a toujours été culturel, même quand les motivations principales des touristes ont évolué au cours du siècle dernier pour intégrer les loisirs, le divertissement ou encore le repos que ceux-ci recherchent dans les stations balnéaires par exemple. Cette notion a donc fait l'objet de très nombreuses définitions, chaque auteur proposant une interprétation qui convient à ses objectifs, ses perspectives ou ses besoins. Dès lors, la recherche d'une définition peut effectivement sembler une « impasse intellectuelle [qui] implique un jugement de valeur $a$ priori sur ce qui relève ou non de la culture ou du culturel » (Cousin, 2008 : 42). Pourtant, le succès de cette notion est sans doute révélateur du contexte dans lequel elle émerge et se développe, celui de la croissance exponentielle du tourisme dans la seconde moitié du XXe siècle.

12 De plus, la communauté internationale montre aussi à ce moment un intérêt croissant pour l'environnement humain, avec la conférence des Nations Unies sur l'environnement à Stockholm en 1972, et pour le patrimoine culturel et naturel avec l'adoption de la convention concernant la protection du patrimoine mondial, culturel et naturel à l'Unesco, en 1972 également. Ce contexte général favorise donc l'émergence de la notion de tourisme culturel à partir des années soixantedix, à laquelle nous nous intéressons ici. La charte du tourisme culturel, adoptée par Icomos en 1976, définit le tourisme culturel comme « celui qui a pour objet, entre autres objectifs, la découverte des sites et des monuments » (Icomos, 1976). Ce tourisme culturel qui apparaît et qui s'intéresse notamment au patrimoine ne représente donc qu'une " niche marketing » concernant surtout les visites du patrimoine monumental. Cependant, les notions de culture et de patrimoine se sont élargies pour inclure de nombreuses autres pratiques - artistiques, gastronomiques, festives, etc. Toujours dans une approche plutôt opérationnelle, ceci a conduit de nombreux chercheurs à proposer des typologies de touristes, démarche cohérente avec la préoccupation économique qu'elle sous-entend. On retrouve donc des catégories élaborées en fonction des activités culturelles impliquées (Johan \& Joppe, 2005), en fonction des motivations des touristes (McKercher \& du Cros, 2003 ; Patin, 1988), voire des deux (Lutz, 2005, cité dans Chevrier, 2006).

13 Cependant, cet élargissement conjoint des notions de patrimoine et de culture a également rendu difficile, voire impossible pour le chercheur, de produire une définition qui permette de rendre compte des phénomènes observés et étudiés, provoquant « l'impasse intellectuelle » évoquée plus haut. La complexité croissante de la notion de patrimoine, notamment lorsqu'il est conçu comme une construction sociale, a amené les chercheurs à autonomiser le phénomène du tourisme patrimonial pour pouvoir étudier des pratiques nouvelles dans lesquelles se développent de nouveaux modes d'interaction entre les touristes, les communautés locales et le patrimoine.

14 Ces nouveaux types de relations se sont multipliés dans les dernières décennies autour de notions « nouvelles », telles que le développement durable, et de pratiques particulières, comme par exemple le « tourisme des racines » (Fourcade, 2010) où le touriste recherche sa propre histoire et « son » patrimoine. Cette question de l'appartenance du patrimoine prend d'ailleurs une importance notable dans le cas du tourisme patrimonial. En effet, le patrimoine n'existe pas sans quelqu'un qui le revendique comme tel. Autrement dit, le patrimoine est toujours celui d'un groupe en particulier, duquel certains individus font partie et d'autres sont exclus. Dans ce cas, on peut se demander si le tourisme patrimonial ne devrait pas plutôt se définir à partir de ce que les touristes, plutôt que les communautés locales, considèrent comme étant du patrimoine. Certains auteurs ont proposé une définition centrée sur les motivations et les perceptions des visiteurs plutôt que sur les caractéristiques du site (Poria et al., 2001 et 2003). Dans ce cas, c'est la connaissance par le touriste de ce qui devrait être, selon lui, du patrimoine qui lui permet de reconnaître un site ou un élément en tant que tel. Par exemple, les visiteurs européens auront des difficultés à reconnaître des éléments patrimoniaux nouvellement construits sous prétexte qu'il s'agit en fait d'une pratique rituelle, comme c'est le cas parfois avec certains édifices religieux en Asie, ou alors ils se référeront au « patrimoine immatériel » dont la définition et la sauvegarde posent encore de nombreuses questions. Dans tous les cas, ils préféreront sans doute les grands monuments, qui sont en adéquation avec leur propre compréhension du patrimoine. Dans cette approche, fondée sur la perception et les motivations des touristes, Yaniv Poria et al. distinguent trois types de touristes patrimoniaux. Selon eux, cela peut désigner ceux qui visitent des sites qu'ils 
considèrent patrimoniaux bien qu'ils ne les relient pas à leur propre patrimoine, ceux qui visitent des sites qui ne sont pas nécessairement reconnus localement comme patrimoniaux mais que les touristes considèrent comme faisant partie de leur patrimoine, et ceux qui visitent des sites reconnus comme patrimoniaux mais qui ne sont pas informés de cette reconnaissance (Poria et al., 2001). Ces définitions leur permettent par ailleurs de différencier le tourisme patrimonial de ce qu'ils nomment le « tourisme historique », lequel désigne les visites motivées par les attributs historiques du site.

15 Ces travaux sur le tourisme patrimonial, et l'autonomisation de cet objet d'étude, sont également la conséquence de la transformation du contexte global des échanges dans lequel s'inscrit le tourisme. Comme les autres flux de personnes et de marchandises, le tourisme s'est internationalisé à tel point que les arrivées internationales ont dépassé, à l'échelle mondiale, le 1,1 milliard en 2013 (OMT, 2014). Cette dimension internationale du tourisme touche aussi le tourisme patrimonial, ce qui crée des différences entre les touristes nationaux et internationaux au moment de reconnaitre ou non un site visité comme étant patrimonial. En effet, il va de soi qu'il sera plus évident dans le cas du tourisme national que la visite de sites reconnus légalement comme patrimoniaux corresponde à ce que les touristes eux-mêmes considèrent comme étant leur patrimoine (Park, 2010). Dans le cas des touristes internationaux, la situation est plus complexe quoique le développement du patrimoine mondial ces quatre dernières décennies ait pu justement contribuer à l'apparition de codes et de pratiques généralisés, qui dépassent les cultures et les coutumes des visiteurs et des visités. Le tourisme en général, et patrimonial en particulier, reposerait donc sur la capacité des touristes à reconnaître des lieux, des pratiques ou des cultures qui correspondent à leurs attentes - en y répondant ou en les déjouant - ou à des éléments qu'ils connaissent déjà. Dans cette perspective, la mise en tourisme du patrimoine culturel consiste alors à établir un lien entre l'expérience vécue par le touriste et ses connaissances, expériences et attentes préalables. On comprend alors mieux l'enjeu de la mise en tourisme du patrimoine lorsqu'elle s'adresse à des touristes d'origines si différentes et pourquoi, comme on le verra plus loin, la prise en compte de l'origine et des attentes potentielles des touristes peut influencer non seulement la mise en tourisme, mais aussi la signification même du patrimoine.

\section{Tourisme et patrimoine mondial}

Le patrimoine mondial a connu une évolution notable depuis l'adoption de la convention concernant la protection du patrimoine mondial, culturel et naturel. Le projet initial n'avait que peu à voir avec le tourisme, ni même avec les cultures locales, puisqu'il s'agissait avant tout de préserver des sites ayant une «valeur universelle exceptionnelle ». Bien que le tourisme soit déjà présent dans les préoccupations d'organismes internationaux comme l'Icomos, le rapport avec le patrimoine se construit sur un discours d'opposition (Cousin, $2008: 42$ ). Ce n'est qu'à la fin des années quatre-vingt-dix que les évolutions, tant du patrimoine mondial que du tourisme, conduiront à un rapprochement. D'une part, le patrimoine mondial connait une forte croissance et s'ouvre à l'importance des communautés locales dont le développement passe souvent par une exploitation économique des ressources patrimoniales. D'autre part, le tourisme se transforme en l'une des principales activités d'échanges internationaux pour devenir une «industrie [...] à la fois polyfonctionnelle et globale » (Lanfant, $2004: 370$ ). Comme on l'a vu ci-dessus, la contradiction qui semblait séparer tourisme et patrimoine se résout à la fois dans l'élargissement de la notion de culture vers celle de diversité culturelle et dans l'élargissement et l'autonomisation de la notion de patrimoine, mais aussi dans la généralisation des principes de développement durable des communautés et des sites, qu'ils soient réellement applicables ou non.

17 De plus, la préséance semble s'être inversée au début des années 2000 et plutôt qu'un privilège accordé aux quelques « touristes culturels » intéressés, le patrimoine semblerait maintenant contraint de s'adapter aux activités touristiques, sous peine de n'être plus tout à fait patrimonial car empêchant sa reconnaissance et son appropriation par le visiteur, c'est-à-dire ce que certains chercheurs définissent comme " tourisme patrimonial ». Au bout du compte, la patrimonialisation vient explicitement, comme nous le verrons cidessous dans le cas d'Arequipa, servir le développement de l'attractivité touristique. Dans cette perspective, on peut effectivement se demander si le patrimoine n'est pas « dépendant » du tourisme et si, comme le suggère Saskia Cousin, le tourisme, plus que le patrimoine, n'est pas devenu le " nouveau cadre de légitimation » de l'Unesco (Cousin, 2008 : 52), et plus généralement d'autres politiques internationales. D'ailleurs, le Centre du patrimoine mondial de l'Unesco s'est récemment impliqué dans la question du tourisme, indirectement d'abord par la signature d'un accord de collaboration avec l'entreprise TripAdvisor en 2009, puis directement avec la mise en place du programme sur le patrimoine mondial et le tourisme durable en 2011. Ce changement de paradigme dans les relations entre tourisme et patrimoine semble se confirmer à la lecture des recommandations d'Assouan en vue d'optimiser le rôle du tourisme dans le rapprochement des cultures, adoptées lors de la journée mondiale du tourisme de 2011, et selon lesquelles les professionnels du tourisme agissent comme «médiateurs entre les visiteurs et les communautés locales ", tout en suggérant de " rendre obligatoire l'engagement de la communauté locale dans le développement du tourisme » (OMT, 2011). Il semble donc s'effectuer un glissement vers une situation où la culture, dont le patrimoine, n'est plus simplement un produit que l'on peut intégrer à l'offre touristique, mais où c'est plutôt le tourisme qui sert de médiateur à la culture.

18 Dès lors, on peut se demander dans quelle mesure les transformations d'un site patrimonial sont influencées par les représentations sociales associées au tourisme, particulièrement dans un site inscrit au patrimoine mondial. En d'autres termes, si les représentations sociales ont un impact sur les transformations physiques du site, quelle est l'influence des représentations construites ou transformées par les professionnels du tourisme ? On pourrait alors se questionner sur leur rôle dans la patrimonialisation et se demander si la place particulière du tourisme dans les sites inscrits au patrimoine mondial relève des effets de cette inscription, tels que l'on a pu les mettre en évidence (Dormaels, 2013), ou si le tourisme induit des transformations physiques et symboliques du site. Tenter de répondre à ces interrogations pourrait conduire à remettre en question la façon dont on envisage le patrimoine mondial.

\section{Le centre historique de la ville d'Arequipa}

19 Alors que peu d'études ont abordé la patrimonialisation des sites urbains du patrimoine mondial, celles-ci n'analysent pas la relation que ce processus peut avoir avec le phénomène touristique. La littérature fait référence à la coexistence et aux enjeux contradictoires de préservation pour l'un et de développement pour l'autre (Evans, 2002), particulièrement 
dans le cadre du patrimoine urbain (Pendlebury et al., 2009), ou encore au lien empirique qui existe entre patrimoine et tourisme, et peu aux relations conceptuelles entre les processus et dans leur évolution. Notre objectif est donc de contribuer à une meilleure compréhension du rôle du phénomène touristique dans la construction des représentations qui constituent le caractère patrimonial des sites urbains inscrits au patrimoine mondial.

20 Cette étude est issue de notre recherche doctorale et de son prolongement dans un projet de recherche postdoctorale. Elle repose sur une approche historico-interprétative (Groat \& Wang, 2002 ; Morisset, 2011) et a consisté en l'analyse et l'interprétation de données collectées selon quatre méthodes : les questionnaires, les entretiens semi-dirigés, les recherches documentaires et l'observation directe. Ces différentes sources ont permis de corroborer et préciser les données obtenues. Dans notre recherche antérieure, l'analyse comparative de documents iconographiques de différentes époques - images, plans, etc. -, ainsi que l'observation directe, ont permis de comprendre les évolutions de l'espace urbain et les significations attribuées au patrimoine par les institutions locales et les habitants. Pour cette étude, nous avons repris certaines de ces données et nous avons également étudié les produits touristiques, notamment les circuits de visite organisés qui sont offerts par la plupart des agences de la ville. Deux méthodes ont été utilisées pour analyser ces données, à savoir la codification thématique des questionnaires et l'analyse inductive du contenu des entrevues et des documents colligés - notamment la presse locale et les guides touristiques -, mais aussi des produits touristiques proposés. Cela a permis de mettre en évidence les représentations sociales construites ou influencées par les professionnels du tourisme, ainsi que leur évolution.

21 L'analyse interprétative de ces éléments, espaces urbains et signification symbolique d'une part, et mise en tourisme et représentations sociales des professionnels du tourisme d'autre part, a permis la reconstruction herméneutique des deux processus, celui de la patrimonialisation du site (Dormaels, 2013) et celui de sa mise en tourisme. En effet, dans le cas du patrimoine urbain, on peut documenter et analyser les étapes de la patrimonialisation au travers de l'évolution de l'environnement urbain - plans, photographies, cartes postales, observation - et des représentations qu'en ont ceux qui y vivent - publications associatives, articles de presse, entretiens et questionnaires, documents de planification, rapports sur l'état de conservation, etc. De façon analogue, dans ces documents et d'autres - études de fréquentation, profil des touristes, etc. -, on peut retrouver ces mêmes jalons de la construction de l'attraction touristique. Enfin, dans les deux cas, ces données ont été complétées par des entretiens et des questionnaires. Ceux-ci ne peuvent bien sûr servir qu'à mettre en évidence les représentations au moment où ils sont menés, mais ils permettent aussi de mettre en lumière certaines dynamiques entre les acteurs - habitants, touristes, entreprises de l'industrie touristique, institutions publiques, etc. -, qui, si elles ne font pas directement l'objet de cet article, contribuent à la compréhension et à l'interprétation des représentations sociales et des transformations physiques et sociales du centre-ville. Lors de nos recherches, nous avons interrogé par questionnaire des habitants et des touristes - vingt-huit habitants et dix touristes et mené treize entretiens semi-dirigés, d'une durée d'une heure à une heure trente, avec des personnes dans les administrations impliquées dans la gestion et l'inscription du site et dans sa mise en tourisme. Dans l'analyse notamment dans la codification thématique - de ces treize entretiens, on a distingué ce qui relève de la position «officielle » de l'institution représentée, corroborée par des documents officiels - politiques, rapports, études, etc. -, et ce qui relève de l'interprétation de chaque individu en fonction de ses intérêts et qui contribue à la compréhension des représentations sociales associées au patrimoine et au tourisme. Enfin, précisons que l'objectif ici n'est pas tant d'expliquer les motivations des différents acteurs à agir pour le développement du patrimoine ou du tourisme que de mettre en évidence les influences réciproques des phénomènes touristiques et patrimoniaux, et leurs évolutions dans le temps. La diversité des méthodes nous a permis d'identifier et de documenter les différentes significations patrimoniales, qui ont fait l'objet d'une médiation pour les touristes, depuis les années soixante et particulièrement entre 1999 et 2011.

\section{L'offre touristique du site}

Le lien entre le patrimoine mondial et une « forte fréquentation touristique », qui reste à démontrer dans bien des cas, semble aujourd'hui devenu un lieu commun. Pourtant, la fréquentation touristique d'un site inscrit dépend aussi d'autres facteurs, parmi lesquels sa notoriété (Rátz \& Puczkó, 2003 : 471 ; Florent, 2011). Sur l'ensemble des sites inscrits au patrimoine mondial, une majorité ne connait pas de problème de surfréquentation, et certains semblent expérimenter une situation enviable où le tourisme est suffisamment important pour être un atout économique sans saturer la capacité des infrastructures et des attractions. Cet équilibre entre préservation du patrimoine, amélioration de la qualité de vie des habitants et développement économique par le tourisme, que souhaitent atteindre de nombreux gestionnaires, est cependant bien fragile. Toutefois, il apparaît clairement, et notre étude le montrait (Dormaels, 2013), qu'une telle situation est plus probable lorsque le développement touristique a motivé la mise en valeur du patrimoine. Dans le cas d'Arequipa, on le verra, il s'agit en effet de ce que O. Lazzarotti définit comme une « synergie » puisque le patrimoine et le tourisme sont « activés ensemble pour être orientés vers un but commun » (Lazzarotti, 2003 : 97). Au Pérou, parmi les touristes étrangers qui mentionnent le tourisme culturel comme motivation de voyage, $99 \%$ citent le tourisme urbain (Promperu, 2010 : 15). Par ailleurs, 23 \% des touristes étrangers en vacances visitent Arequipa (Promperu, $2010: 13$ ). Bien que de nombreux touristes viennent à Arequipa pour d'autres raisons que la ville elle-même, elle est tout de même visitée par $85 \%$ d'entre eux. Cependant, les touristes sont surtout " de passage » à Arequipa et la durée moyenne du séjour des touristes internationaux en 2012 était de 1,6 jour (Mincetur, 2013, données compilées par l'auteur). On comprend donc que le centre historique et les nombreux monuments et musées ne soient que très rarement extensivement visités. Au contraire, les touristes vont plutôt à « l'essentiel ».

23 L'une des principales raisons expliquant cette durée de séjour est la proximité d'une attraction touristique plus importante que le centre historique : le canyon de Colca. Cette formation naturelle et son voisin le canyon de Cotahuasi sont les canyons les plus profonds du monde et le Colca est l'une des attractions touristiques majeures du sud du pays, avec les lignes de Nazca et le lac Titicaca. De nombreux touristes viennent dans cette région pour effectuer une randonnée dans le canyon afin de profiter des panoramas, découvrir des ruines archéologiques et des peintures rupestres, et observer le vol des condors qui nichent dans cette région. Cependant, le canyon est situé dans une région peu développée et Arequipa est la ville la plus proche disposant d’infrastructures touristiques suffisantes, et notamment l'aéroport international et la gare d'autobus, pour accueillir les touristes. On peut donc considérer que tous les touristes qui souhaitent visiter le Colca passent par Arequipa puisque c'est de la ville que partent les excursions organisées par les 
agences locales. Or, les chiffres de fréquentation fournis par le ministère du Commerce extérieur et du Tourisme du Pérou (Mincetur) indiquent presque le même nombre de visiteurs étrangers pour le Colca (119 605 en 2011 et 116012 en 2012, contre 76484 touristes nationaux en 2012) que pour le monastère Santa Catalina d'Arequipa (123 983 en 2011 et 125305 en 2012, contre 48476 touristes nationaux en 2012) (Mincetur, 2013). Le centre-ville historique n'est donc pas la motivation principale, ou à tout le moins pas la seule, des touristes qui viennent à Arequipa, mais le patrimoine reste une attraction majeure. On se trouve donc dans une situation complexe de tourisme impliquant différentes pratiques en ville, qui relèvent du tourisme patrimonial tel que nous l'avons précisé plus haut. En effet, les touristes majoritairement étrangers perçoivent le patrimoine de la ville selon leur propre expérience et leur culture, à travers les visites de monuments, mais aussi la gastronomie ou les traditions locales.

24 Cependant, le développement touristique de la ville ne date pas de son inscription au patrimoine mondial puisque dès 1940 était construit un hôtel de luxe, l'hôtel « de turistas » ( des touristes »). C'est surtout dans les années soixante-dix que de nombreux investissements ont eu lieu afin de créer des attractions touristiques, notamment avec la constitution en août 1970 de l'entreprise Promociones Turísticas del Sur SA dédiée à la promotion touristique et chargée, entre autres, de la restauration du monastère Santa Catalina pour en permettre l'exploitation touristique. Ce n'est pas la seule attraction touristique qui verra le jour au cours de cette décennie puisque le Banco Central Hipotecario, une institution bancaire, s'implique dans la restauration de plusieurs édifices patrimoniaux, dont les cloîtres de l'église de La Compañía, transformés en galerie commerciale en 1971, et le moulin de Sabandía, inauguré en septembre 1973. Sans oublier un autre important couvent, La Recoleta, qui se transforme en musée en 1978 afin d'exposer les collections anthropologiques, zoologiques et artistiques rassemblées par les missionnaires franciscains, ainsi que leur bibliothèque d'environ 20000 volumes. Ces attractions figurent aujourd'hui encore en haut de la liste des sites visités à Arequipa. C'est donc véritablement dans les années soixante-dix que commence à se faire sentir l'influence du tourisme dans la restauration de certains bâtiments et la patrimonialisation du centre-ville. Comme l'a montré notre recherche (Dormaels 2013), l'attractivité touristique d'Arequipa n'a pas connu de modification majeure entre cette période et la fin des années quatrevingt-dix où l'on intervient de nouveau sur l'espace urbain pour permettre l'inscription sur la Liste du patrimoine mondial et attirer une nouvelle clientèle touristique. Cependant, on passe pendant cette période d'un ensemble de monuments distincts à l'existence d'un centre historique, notamment avec la création d'une "zone monumentale » protégée en 1972. D’ailleurs, notre étude de la patrimonialisation du centre historique montre que le site était déjà une attraction patrimoniale avant son inscription, comme en témoigne un plan touristique de 1990, mais d'une taille plus petite et limité aux abords de la place principale.

25 En revanche, des changements majeurs s'opèrent à partir de 1999 puisque l'inscription devient un projet sur lequel le maire récemment élu a fait campagne. La volonté, exprimée clairement dans le dossier de candidature du site, était alors de valoriser le patrimoine pour relancer l'économie touristique locale et en faire un outil de développement et de création d'emplois. De ce point de vue, on pourrait considérer que l'objectif a été atteint puisque le nombre total de touristes est passé de 81328 en 2001 à 167114 en 2011² (Mincetur, 2013). De plus, selon le journal El Comercio, l'investissement dans les infrastructures hôtelières aurait atteint trente millions de dollars US entre 2006 et 2009 seulement (El Comercio, 2009). On constate d'ailleurs la croissance du secteur hôtelier puisque la capacité, pour la ville d'Arequipa, est passée de 95 établissements pour 1727 chambres et 2987 places en 2001 à 403 établissements pour 7189 chambres et 13399 places en 2011 (Mincetur, 2011). Bien que certains de ces établissements ne soient pas situés dans le centre-ville, il reste néanmoins que leur multiplication est révélatrice de la croissance du tourisme. Toutefois, comme on l'a vu plus haut, ce succès est relatif si l'on considère le temps de séjour encore assez court et symptomatique d'une attractivité du centreville relativement limitée face à la concurrence des autres sites de la région - Colca - et des autres sites culturels du pays : Cusco, Machu Picchu, Nazca, etc.

26 Au-delà de la capacité hôtelière, cette multiplication des infrastructures a évidemment un impact sur le paysage urbain, transformant et agrandissant de nombreux édifices devenus hôtels, au détriment du logement. Mais cette transformation de l'espace urbain va plus loin que le seul aménagement d'infrastructures et concerne aussi les espaces publics. En effet, depuis 1999, la municipalité a entrepris des travaux de restauration du centre-ville, qui font partie des projets inscrits dans le plan directeur déposé en 2002. Ceux-ci, répartis en douze programmes distincts, concernent aussi bien la réfection de la voirie et des trottoirs, l'enterrement des réseaux, la réhabilitation des places publiques que la formation du personnel municipal, le développement touristique ou encore la sécurité. L’analyse de ces projets permet de souligner plusieurs aspects et particulièrement deux caractéristiques qui sont, selon nous, directement liées à l'inscription et au développement de l'attractivité touristique du site. D’une part, les transformations privilégient la circulation des piétons avec la création de trottoirs élargis, qui n'existaient pas, et la piétonisation de deux rues du centre-ville.

$27 \quad$ Si ces interventions contribuent à l'amélioration de la qualité de vie des résidents, leur localisation révèle qu'elles sont aussi destinées au tourisme car les premières rues aménagées sont celles qui partent de la place principale - place d'Armes -, qui sont surtout empruntées par les touristes et où se situent le plus grand nombre d'hôtels, de restaurants et de boutiques de souvenirs. D’autre part, les interventions menées ont mis l'accent sur les caractéristiques qui ont justifié l'inscription sur la Liste du patrimoine mondial. Ainsi, on remarque par exemple que la restauration du quartier San Lázaro met en avant le matériau local de construction, le « sillar » blanc - un tuf volcanique -, qui, laissé à nu, renvoie à une représentation d'Arequipa en tant que « ville blanche » largement diffusée, sinon construite, par la communication touristique. En outre, le quartier a fait aussi l'objet d'une piétonisation partielle, le tout afin de répondre au changement de vocation souhaité dans le plan d'occupation des sols, intégré au plan directeur, qui proposait de développer l'usage commercial touristique de ce secteur surtout résidentiel. Ces deux exemples illustrent comment les interventions sur les espaces publics peuvent être des aménagements qui ne renvoient pas toujours à une réalité historique, mais bien aux besoins d'un centre historique touristique, tout en prenant soin de créer une cohérence esthétique par l'utilisation de matériaux traditionnels entre autres. Pour nous, comme nous le verrons plus bas, ces interventions constituent la première et la principale action de médiation du centre historique d'Arequipa.

28 Toutefois, ces transformations ont aussi un impact sur l'environnement immédiat de la ville. D'abord, l'augmentation du tourisme génère un accroissement du volume de transport et des problèmes associés de circulation et de pollution. Mais surtout, la protection du centre historique limite les développements immobiliers et fonciers, ce qui, avec l'augmentation des hôtels et restaurants, contribue à repousser les autres usages, notamment le résidentiel, en périphérie. Ces effets, combinés aux autres facteurs d'accroissement de la population, ont créé une pression sur les zones immédiatement autour de la ville, semi-urbanisées, et qui sont surtout composées de cultures en terrasses précoloniales, 
d'où le nom de « campagne » (« campiña »). Ces zones sont d'autant plus importantes puisqu'il s'agit d'une région aride : elles peuvent difficilement être déplacées et elles semblent avoir un effet de tempérance sur le climat local. Il y a donc une préoccupation croissante à l'égard de ces zones, cohérente aussi avec une préoccupation globalement grandissante pour le développement durable et l'environnement. Cet intérêt se manifeste notamment dans l'évolution des représentations associées à cette « campagne » et son intégration dans les offres touristiques et dans la gestion du patrimoine.

\section{Les représentations touristiques}

L'analyse de l'offre touristique et des représentations des professionnels du secteur, dans le cas d'Arequipa, permet de mettre en évidence leurs liens avec les représentations patrimoniales exprimées dans l'énoncé de valeur universelle exceptionnelle du site. En effet, le centre historique a été inscrit sur la Liste du patrimoine mondial au titre des critères i et iv pour :

« Critère i : L'architecture ornementée du centre historique d'Arequipa est un chef-d'œuvre qui illustre la fusion créative des caractéristiques européennes et autochtones, qui a joué un rôle crucial dans l'expression culturelle de toute la région.

Critère iv : Le centre historique d'Arequipa est un exemple exceptionnel de peuplement colonial caractérisé par les conditions naturelles, les influences indigènes, la conquête et l'évangélisation, ainsi que par son environnement naturel spectaculaire. » (Unesco, 2013.)

L'exceptionnalité d'Arequipa, au sens du patrimoine mondial, repose donc sur son caractère de mixité culturelle qui se traduit dans un style architectural particulier, appelé « arquitectura mestizada » (« architecture métissée ») proche du «barroco andino » (« baroque andin »), mais aussi dans le mélange produit par la colonisation, l'influence indigène et les conditions naturelles, qui renvoient principalement au climat sec et aux séismes - courants dans la région. L'industrie touristique a depuis repris largement ce discours, comme on peut le constater dans la description offerte par le Guide Vert Michelin en ligne :

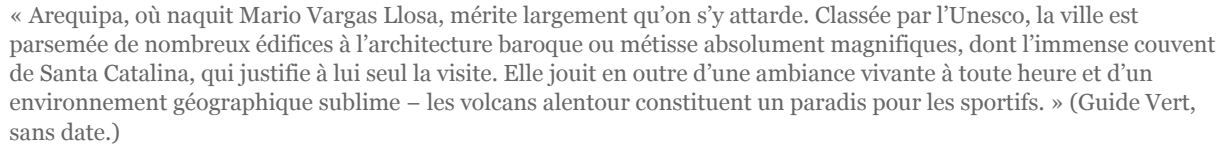

Le guide conseille sept attraits touristiques : le monastère Santa Catalina, l'église de La Compañía, le Museo Santuarios andinos, le Museo de Arte virreinal Santa Teresa, la place d'Armes, la cathédrale d'Arequipa et la vieille ville d'Arequipa. Le site spécialisé TripAdvisor insiste, lui, dans sa description de la ville, sur l'image de « ville blanche » et sur les attraits architecturaux :

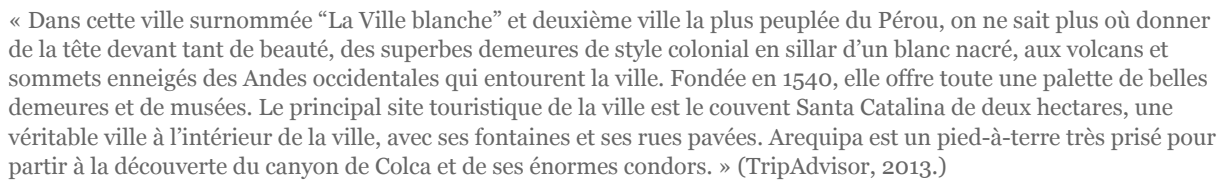

De son côté, le Guide du Routard (2013) recommande également sept attraits à voir : la Casa del Moral - une demeure coloniale du XVIIIe siècle -, le monastère Santa Catalina, l'église de San Francisco, l'église de La Compañia, le monastère de Santa Teresa, la place d'Armes et le volcan Misti. Cependant, il consacre également une page de son site au seul canyon de Colca. D'ailleurs, si cette sélection des guides met clairement l'accent sur le patrimoine bâti, les produits touristiques les plus offerts sur place concernent d'autres attractions touristiques (Pertur, 2010 : 22), au premier rang desquelles le canyon de Colca, mais aussi beaucoup d'autres qui ne sont pas dans le périmètre de la zone centrale inscrite au patrimoine mondial, ni même dans la ville, mais plutôt dans ses alentours - Carmen Alto, Yanahuara, Sachaca, Tingo, Paucarpata, moulin de Sabandía, Mansión del Fundador -, c'est-à-dire dans la campiña. Ainsi, il y a localement une mise en tourisme importante des attractions naturelles et pas seulement culturelles.

33 Nous voyons donc à travers ces exemples comment les discours de l'industrie touristique reprennent à leur compte des éléments qui justifient l'inscription du site au patrimoine mondial. Toutefois, on trouve aussi des représentations antérieures à l'inscription, comme celle de la «Ville blanche » déjà évoquée, surnom que l'industrie touristique a sans doute contribué à populariser ${ }^{3}$. En effet, celle-ci se sert abondamment de cette dénomination et de l'imaginaire qu'elle convoque pour communiquer aux touristes potentiels la représentation d'une ville ancienne et monumentale, à l'image à la fois des anciens édifices et de la cathédrale. Nous retrouvons aussi, dans de nombreux documents, l'image d'une ville ancienne, coloniale et patrimoniale. Qu'il s'agisse d'articles de presse ou de documentation touristique, les illustrations de la ville et de son centre historique convoquent les mêmes éléments visuels que sont la cathédrale, les monastères, les couvents et les maisons du XVIIIe siècle. Ainsi, le dépliant-plan officiel édité par iPeru, l'office de tourisme national, présente en couverture la façade de la cathédrale et à l'intérieur une vue du monastère Santa Catalina. La communication à destination du public touristique insiste généralement sur ces deux édifices qui sont les plus visités, et notamment le monastère Santa Catalina considéré comme exceptionnel. Les deux se retrouvent aussi dans les illustrations du guide Lonely Planet «Peru » de 2000 (Rachowiecki, 2000), et le monastère est le troisième des huit lieux « classiques » de visite dans le guide Lonely Planet « Pérou » de 2010 (Miranda et al., 2010). Toutefois, la façade de la cathédrale semble plus généralement le symbole de la ville historique et de son centre. Les images à destination des touristes servent donc non seulement à produire une impression de familiarité pour les touristes, lesquels trouvent là une monumentalité qui signifie pour eux qu'il s'agit d'un patrimoine remarquable, mais aussi à illustrer le discours construit sur les éléments de justification de l'inscription du site.

\section{La médiation du patrimoine à Arequipa}




\section{Les outils de médiation du patrimoine}

34 Dans le centre-ville d'Arequipa, on trouve assez peu d'outils de médiation du patrimoine. Celui qui est le plus présent et que l'on retrouve affiché une quinzaine de fois est le plan touristique du centre historique. Il est notable que, comparativement au plan de 1990 qui se limitait à quelques rues autour de la place centrale et du monastère Santa Catalina, cette version plus récente reprend exactement les limites de la zone tampon inscrite au patrimoine mondial. En effet, lors de l'inscription sur la Liste, les sites doivent être clairement délimités et divisés en deux zones : la zone centrale, qui fait l'objet de l'inscription et porte la valeur universelle exceptionnelle, et la zone tampon, qui l'entoure et permet de protéger la zone centrale en minimisant les impacts du développement du reste de la ville sur celle-ci. Le centre historique d'Arequipa, d'après ces plans, n'est donc plus limité aux quelques monuments principaux, ni même à la zone centrale du site inscrit, mais recouvre plutôt une superficie plus large qui intègre presque toute la ville construite avant 1950. Ceci a provoqué un glissement du statut patrimonial de ces secteurs car initialement, ils n'étaient pas considérés de première importance puisqu'ils n'avaient pas été inclus dans la zone centrale inscrite en 2000. Mais leur statut a évolué, notamment par l'influence des représentations produites par les professionnels du tourisme et que l'on retrouve dans les plans que chaque hôtel distribue à ses clients et qui sont affichés sur les murs de la ville. Cette évolution se manifeste aujourd'hui notamment dans le projet d'extension des limites du site inscrit qui, précisément, transforme la zone tampon en zone centrale et crée une nouvelle zone tampon élargie. Bien que cette redéfinition corresponde aussi à d'autres objectifs, cette reprise des limites exactes nous semble significative de l'extension de la signification patrimoniale par l'influence d'une représentation touristique, dans ce cas d'abord graphique avant que sociale.

35 Il semble également que cette évolution corresponde à un changement plus général de la notion de patrimoine mondial, notamment avec l'intégration de nouvelles valeurs patrimoniales liées aux paysages et à l'importance du développement durable. On observe par exemple à Arequipa, comme dans d'autres sites, l'existence d'un « effet Dresde » (Dormaels, 2013), c'est-à-dire que les enjeux de conservation, surtout environnementale, servent d'argument contre certains développements qui menaceraient la valeur patrimoniale du site et son maintien sur la Liste du patrimoine mondial. En effet, ceux qui utilisent ces arguments évoquent souvent le cas du site de la vallée de l'Elbe à Dresde, en Allemagne, inscrit en 2004 et retiré de la Liste du patrimoine mondial en 2009 à cause de la construction d'un pont autoroutier qui porte atteinte à la valeur universelle exceptionnelle du site. Dans le cas d'Arequipa, la construction d'un pont, le pont Chilina, a été et est encore l'objet de débats dans lesquels le risque de retrait de l'inscription est, sans véritable justification, souvent évoqué. L’importance des questions environnementales apparaît donc aussi dans cette redéfinition des limites du site inscrit, laquelle modifie la signification symbolique du centre historique, car il devient un élément « urbain » - colonial - signifiant en regard d’un élément de « nature paysagée » ou « agriculturel » que sont les cultures en terrasse - précoloniales. Le patrimoine est alors envisagé plus comme un ensemble, ou un paysage, composé de la ville, de la campiña et de l'environnement naturel plus large symbolisé par le volcan Misti, très visible depuis la ville. Cette conception du site correspond donc mieux, d'une part à l'évolution de la notion de patrimoine au niveau international, et d'autre part à l'intérêt de nombre de touristes qui viennent pour les richesses naturelles et paysagères de la région, et du Colca en particulier.

36 Toutefois, ce sens est difficilement accessible pour ceux qui ne visitent que la ville pendant leur court séjour car à part les plans, on ne trouve pas d'autres panneaux d'interprétation, dans l'espace public, qui soient destinés aux touristes exclusivement. Les autres sources d'informations disponibles sur place pour les visiteurs se résument aux plans et brochures fournis par les hôtels et les bureaux d'informations touristiques, et aux nombreuses plaques commémoratives qui rappellent les diverses interventions sur les places et les édifices. Enfin, il est possible d'effectuer des visites guidées du centre-ville, mais ce type de produit est le moins vendu des circuits organisés (Pertur, 2010). Cette rareté des outils de médiation s'explique selon nous par deux facteurs. D'une part, on l'a dit, les touristes restent peu de temps à Arequipa. La ville constitue une étape dans le « circuit sud » du Pérou où l'on passe depuis Nazca vers le lac Titicaca, ou pour aller visiter le canyon de Colca. Durant leur séjour à Arequipa, les pratiques de ces touristes sont donc moins tournées vers la découverte minutieuse du patrimoine bâti dans le centre historique et plus vers la promenade. Le peu de temps conduit aussi à limiter le nombre de visites à « l'essentiel » ou « l'incontournable », rôle joué dans ce cas par le monastère Santa Catalina.

37 D’ailleurs, les différences de revenus liées aux visites sont très importantes entre celui-ci et les autres attraits touristiques de la ville, et même pour le couvent San Francisco, très proche de celui de Santa Catalina, la communauté religieuse peine à entretenir le bâti avec les bénéfices issus des droits d'entrée. D'autre part, au-delà des pratiques touristiques, c'est aussi parce que ce que l'on cherche à transmettre aux touristes, ce n'est pas tant l'histoire de la ville dont les bâtiments seraient les témoins, et dont la compréhension requerrait un texte d'accompagnement apportant des données historiques. Notre recherche indique plutôt une évolution dans les représentations associées à la mise en tourisme entre les années soixante-dix et les années 2000.

38 Aujourd'hui, c'est plutôt le centre historique même qui se fait outil de médiation d'autres représentations sociales de la ville - co-construite par les acteurs du patrimoine et les professionnels du tourisme - proposées aux touristes. Celles-ci relèvent plutôt ce que devrait être un site inscrit au patrimoine mondial, et une ville dotée d'une culture mixte héritière des passés autochtone et colonial d'après ces acteurs et ces professionnels. Ainsi, ce sont certaines caractéristiques du site qui assurent la médiation de ces représentations, et qui ne renvoient pas à l'histoire du site ou à l'identité locale mais à ce qui en fait « un site du patrimoine mondial » : son insertion dans un environnement naturel valorisé, les aménagements facilitant la visite - trottoirs élargis et secteurs piétons par exemple -, la propreté des espaces publics, la qualité des infrastructures touristiques - hôtels de qualité, boutiques d'artisanat local voire haut de gamme mais aussi de souvenirs, restaurants gastronomiques, etc. --, bref ce qui contribue à procurer au touriste une expérience « à la hauteur » d'un site inscrit au patrimoine mondial. Ces représentations sont particulièrement visibles dans les « images » produites, c'est-àdire la création d'un espace urbain visuellement cohérent avec les représentations sociales et reproduit dans l'iconographie touristique diffusée à travers les guides, les cartes postales et même les photographies prises par les touristes aux endroits indiqués par leurs accompagnateurs locaux et qui se retrouvent sur Internet.

\section{Quelles images pour les touristes et les résidents ?}


39 L'analyse du cas d'Arequipa met en évidence des relations entre l'évolution des représentations sous diverses influences - dont celle du tourisme -, les transformations du site patrimonial et la médiation du patrimoine, et ce, à plusieurs niveaux. En effet, nous avons observé et documenté des modifications de l'espace urbain dont l'objectif n'est pas de restaurer un état antérieur, mais plutôt de correspondre mieux à la représentation de ce que devrait être un site du patrimoine mondial, en faisant ainsi du centre historique un outil de médiation. Ainsi, la mise en valeur de l'architecture baroque, plus encore que celle de l'époque républicaine, la restauration des espaces publics, le développement d'hôtels et de restaurants de luxe ou encore la piétonisation de certaines rues, sont autant d'éléments qui semblent signifier l'exceptionnalité du site et les valeurs pour lesquelles il a été inscrit. Au-delà de cette image, nous voyons donc l'influence déterminante des critères d'inscription du site. En effet, la « mixité » qui justifie l'inscription d'Arequipa, au départ relevée surtout dans l'architecture, a évolué pour intégrer la dialectique « urbain/rural », ce qui concourt également à une révision des limites du site inscrit pour inclure certaines zones vertes. Cette influence de la justification sur l'évolution de l'élément patrimonial et sa signification symbolique se retrouve aussi dans sa médiation puisque cet argument est amplement développé dans la communication touristique, et surtout dans l'iconographie de la ville associant très fréquemment la cathédrale, monument emblématique, au volcan Misti qui domine la ville et incarne la nature et les cultures autochtones.

40 Nous voyons aussi cette influence du tourisme à travers une autre signification que l'on tente de transmettre aux visiteurs. À Arequipa, comme dans d'autres villes du Pérou telles que la capitale Lima, on a voulu «nettoyer » le centreville. En effet, la propreté est l'une des principales préoccupations exprimées dans les publications de regroupements d'habitants, dans la presse et dans les questionnaires. Elle aura conduit à l'installation d'une centaine de poubelles dans les rues du centre en 2008, et à la mise en place en 2009 d'un système de nettoyage quotidien de l'ensemble des rues du centre par 130 employés municipaux. Mais cela concerne aussi la disparition du centre-ville de nombreux marchands ambulants ou de "faux " guides touristiques - considérés ainsi par les habitants car originaires de la campagne environnante. Derrière ces opérations et ce contrôle réside la volonté de démontrer aux étrangers que l'on est capable de conserver et de restaurer un site d'importance mondiale, que l'on " mérite » cette reconnaissance et les « responsabilités » qu'elle suppose, pour reprendre des termes souvent cités dans la presse locale.

41 Enfin, notre étude suggère l'existence d'une autre forme d'influence du tourisme sur l'évolution du site, de ses représentations et de sa médiation. Il semble en effet que, s'agissant d'un site inscrit au patrimoine mondial, les attentes supposées des touristes puissent être déterminantes dans certains choix d'aménagement, comme cela a sans doute été le cas dans la restauration de l'un des quartiers de la ville, le quartier San Lázaro, mais aussi dans le développement d'infrastructures hôtelières nouvelles. Il n'est pas question ici des attentes réelles des touristes, qui sont construites sur les images et discours que ceux-ci peuvent avoir vus/entendus avant leur arrivée - et qui ne font pas l'objet de cette étude -, mais bien d'une représentation de ces attentes par les parties prenantes dans la mise en tourisme du site, c'est-à-dire ce qu'on suppose des attentes des touristes. L'existence locale de cette représentation peut être comprise comme une mise en abyme des représentations sociales touristiques et patrimoniales, ou, autrement dit, une " métareprésentation ». En effet, les représentations véhiculées par les professionnels du tourisme, et en partie aussi par ceux du patrimoine, sont construites par rapport aux attentes supposées des touristes, qui sont elles-mêmes des représentations. C'est pourquoi on parle alors de métareprésentation. Or celles-ci, on l'a vu, ont une incidence sur l'évolution du patrimoine à travers la transformation des valeurs patrimoniales et de leur médiation. En d'autres termes, sous leur influence, les caractéristiques patrimoniales du site se modifient pour intégrer de nouvelles valeurs qui correspondraient à sa reconnaissance en tant que patrimoine mondial, et qui s'expriment dans des aménagements particuliers - piétonisation, propreté, qualité des infrastructures touristiques, etc. Il en résulte alors un type particulier de patrimoine, que nous appelons « méta-patrimoine », et qui serait une construction sociale s'adressant directement aux touristes. Il est d'ailleurs un élément, mis en évidence dans notre étude antérieure, qui semble confirmer cela (Dormaels, 2013). En effet, nous avons constaté que le patrimoine ainsi produit, ou méta-patrimoine, fait l'objet d'une réappropriation de la part des habitants qui deviennent des médiateurs pour les touristes, défendant même l'idée que seuls les habitants auraient la légitimité requise pour faire visiter le centre-ville. On voit donc ici comment est intégré, dans les stratégies d'appropriation des habitants, le fait que le patrimoine produit s'adresse aux touristes plutôt qu'à eux et comment ils adaptent leur rôle à cette situation. Il faut néanmoins souligner que la construction du méta-patrimoine constitue une " couche " supplémentaire de signification qui n'efface pas pour autant complètement les valeurs et significations patrimoniales antérieures, qui font elles aussi toujours l'objet de médiation. Il s'agit plutôt d'une extension des représentations sociales à travers une multiplication des niveaux de signification, et dont la dernière " couche ", le dernier niveau, suscite une relecture différente des significations précédentes.

42 Par ailleurs, dans le cas d'Arequipa, il semble que cette extension ne soit pas limitée à la multiplication des niveaux de signification, mais qu'elle s'exprime aussi dans un processus d'extension géophysique, car ce ne sont plus les différents bâtiments mais bien leur ensemble, le « centre historique », qui porte la nouvelle signification - et même une partie de la nature environnante dans la seconde extension que constitue la révision des limites du site. Dès lors, c'est l'ensemble urbain lui-même qui devient l'outil de médiation touristique de cette nouvelle signification. Cependant, le cas d'Arequipa n'est pas un « cas-type » de centre historique patrimonial et l'on a vu combien l'inscription sur la Liste du patrimoine mondial avait contribué à la production de nouvelles significations - mixité culturelle, importance universelle, dialectique urbain/rural. Il conviendrait donc, d’une part, de voir si ces notions de méta-représentation et de métapatrimoine peuvent effectivement constituer une grille de lecture et un outil d'analyse du patrimoine dans les sites inscrits. D'autre part, il faudrait se demander si cette signification nouvelle émerge aussi dans les sites qui ne sont pas inscrits au patrimoine mondial, ou dans ceux qui le sont, mais dont la mise en tourisme n'était pas initialement explicite - comme c'est le cas à Arequipa et dans de nombreux autres sites récemment inscrits. Cela permettrait alors d'examiner si cette production de signification est liée à une vision de l'inscription au patrimoine mondial plutôt envisagée comme un acteur de la construction de l'attraction touristique, et moins comme une action de conservation du patrimoine, ce qui paraît être une tendance depuis quelques années.

\section{Conclusion}


$43 \quad$ Ainsi, les relations entre le patrimoine et le tourisme sont plus complexes que de simples liens fonctionnels entre attraction patrimoniale et produit touristique. Le cas du centre historique de la ville d'Arequipa nous donne un exemple de l'influence possible du phénomène touristique sur la patrimonialisation d'un site inscrit au patrimoine mondial. Bien que la mise en tourisme ait commencé plusieurs décennies avant l'inscription, les autorités locales l'ont vu dès le départ comme un moyen de relancer l'économie locale par les activités touristiques. Pourtant, la visite du centre historique ne constitue toujours pas la principale motivation des touristes qui arrivent à Arequipa, ni la seule attraction touristique patrimoniale. L'inscription au patrimoine mondial, si elle a peut-être eu un effet sur l'économie, ne semble pas pour autant avoir transformé le centre historique en un site majeur.

44 On comprend alors pourquoi, dans le cas d'Arequipa, cette situation a conduit à la production d'un patrimoine qui corresponde à son image de " patrimoine mondial », tant au niveau des représentations, construites sur l'énoncé de valeur universelle exceptionnelle qui justifie son inscription, qu'au niveau des infrastructures et du paysage élargi à la fois urbain et « agriculturel ». En effet, le site n'étant pas nécessairement perçu comme « incontournable » ou " exceptionnel », les acteurs locaux ont pu souhaiter que celui-ci " sursignifie » son importance, au-delà de sa signification patrimoniale. Pour ce faire, ces acteurs locaux semblent avoir tenté de répondre aux attentes supposées des touristes par l'aménagement du site de façon à ce que ces visiteurs extérieurs comprennent qu'il s'agit d'un site du patrimoine mondial - infrastructures hôtelières nombreuses et de qualité, zones piétonnes, marchés d'artisanat, propreté et entretien des espaces publics, etc. C'est ce phénomène d'influence de la représentation - qu'ont les acteurs locaux de l'attente des touristes sur l'aménagement physique, l'évolution de la signification et les outils de médiation - que nous avons traduit par l'existence de méta-représentations qui produisent un méta-patrimoine. Il conviendrait alors de mettre ces notions à l'épreuve d'autres cas afin d'en vérifier la pertinence, mais aussi de déterminer si ce phénomène est lié aux sites inscrits au patrimoine mondial, voire à ceux inscrits davantage dans une perspective de développement touristique que de conservation patrimoniale.

\section{Bibliographie}

Amirou (Rachid). 2000. Imaginaire du tourisme culturel. Paris : Presses universitaires de France.

Barthes (Roland). 1957. Mythologies. Paris : Éd. du Seuil.

Benhamou (Françoise). 2012. Économie du patrimoine culturel. Paris : La Découverte.

Chesnel (Marc). 2002. Le Tourisme culturel de type urbain : Aménagement et stratégie de mises en valeur. Paris : L'Harmattan.

Chevrier (François-Gilbert). 2006. « Redéfinir le tourisme culturel, c'est aussi redéfinir la culture ! ». Veille tourisme. Publication en ligne : <http://veilletourisme.ca/2006/o6/15/redefinir-le-tourisme-culturel-cest-aussi-redefinir-la-culture/>. Consulté le 2 mars 2013.

Cousin (Saskia). 2008. «L'Unesco et la doctrine du tourisme culturel : Généalogie d’un "bon” tourisme ». Civilisations, 57, p. 41-56. DOI : $10.4000 /$ civilisations.1541

Cousin (Saskia) \& Réau (Bertrand). 2009. Sociologie du tourisme. Paris : La Découverte.

Davallon (Jean). 1991. "Produire les hauts lieux du patrimoine ", p. 85-102, in Des hauts-lieux : La construction sociale de l'exemplarité / sous la direction d'André Micoud \& Marc Abélès. Paris : Edd. du CNRS.

Davallon (Jean). 1992. « Le musée est-il vraiment un média ? ». Publics \& Musées, 2, p. 99-123. DOI : $10.3406 /$ pumus.1992.1017

Davallon (Jean). 2006. Le Don du patrimoine : Une approche communicationnelle de la patrimonialisation. Paris : Lavoisier/Hermès science publications.

Dormaels (Mathieu). 2013. La Construction du patrimoine mondial : Transformations physiques et appropriation locale dans la patrimonialisation du centre-ville historique d'Arequipa, Pérou. Th. Doct., sciences de l'information et de la communication : université du Québec à Montréal / université d'Avignon et des Pays de Vaucluse, Montréal / Avignon.

Drouin (Martin). 2005. Le Combat du patrimoine à Montréal, 1973-2003. Québec : Presses de l'université du Québec.

El Comercio. 2009. «Inversión hotelera en Arequipa fue de US\$ 30 mlls en últimos tres años ». El Comercio. Publication en ligne : <http://elcomercio.pe/economia/357491/noticia-inversion-hotelera-arequipa-fue-us-30-mlls-ultimos-tres-anos >. Consulté le 2 mars 2013 .

Evans (Graeme). 2002. "Living in a World Heritage City : Stakeholders in the Dialectic of the Universal and Particular ". International Journal of Heritage Studies, 8, p. 117-135.

DOI : 10.1080/13527250220143913

Florent (Luc). 2011. « L'utilisation du label Unesco dans la communication touristique : Entre choix et contrainte ». Téoros, 30, p. 1727. DOI : $10.7202 / 1012238 \mathrm{ar}$

Fourcade (Marie-Blanche). 2007. « Tourisme des racines, expériences du retour ». Téoros, 29, p. 3-7.

García Canclini (Néstor). 2007. Culturas híbridas : Estrategias para entrar y salir de la modernidad. Buenos Aires : Paídos. [Première éd. en 1989.]

Gravari-Barbas (Maria), Bourdeau (Laurent) \& Robinson (Mike). 2012. Tourisme et Patrimoine mondial. Québec : Presses de l'université Laval.

Groat (Linda) \& Wang (David). 2002. Architectural Research Methods. New York : J. Wiley.

Guide Vert en ligne. Sans date. " Guide de voyage Arequipa ». Publication en ligne : <http://voyage.michelin.fr/web/destination/Perou-Arequipa >. Consulté le 2 mars 2013.

International Council on Monuments and Sites (Icomos). 1976. Charte du tourisme culturel.

Johan (Novie) \& Joppe (Marion). 2005. « Tourisme culturel et patrimonial : Examen des études de marché existantes ». Ottawa : Gouvernement $d u$ Canada. Publication en ligne : <http://publications.gc.ca/collections/collection_2007/ch-pc/CH52-132005F.pdf >. Consulté le 2 mars 2013.

Knafou (Rémy). 2009. « Préface », p. 11-14, in Culture, Tourisme et Développement : Les voies d'un développement / sous la direction de Claude Origet du Cluzeau \& Jean-Michel Tobelem. Paris : L'Harmattan.

DOI : 10.1016/So070-2161(08)60321-4

Lanfant (Marie-Françoise). 2004. « L'appel à l'éthique et la référence universaliste dans la doctrine officielle du tourisme international ». Tiers-Monde, 45, p. 365-386.

DOI : $10.3406 /$ tiers.2004.5469

Lazarrotti (Olivier). 2000. « Patrimoine et tourisme : Un couple de la mondialisation ». Mappemonde, 57, p. 12-16. 
Lazarrotti (Olivier). 2003. « Tourisme et patrimoine : Ad augusta per angustia ». Annales de Géographie, 112, p. 91-110.

MacCannell (Dean). 1999. The Tourist : A New Theory of the Leisure Class. Berkeley : University of California Press. [Première éd. en 1976.]

Marcotte (Pascale) \& Bourdeau (Laurent). 2008. « L’industrie touristique et l'inscription sur la Liste du patrimoine mondial : Attentes et déceptions ", in Actes de la journée de recherche Pratiques innovantes en matière culturelle et retombées économiques, ESC SaintÉtienne, décembre 2008, Saint-Étienne. Publication en ligne : <http://www.esc-saint-etienne.fr/IMG/pdf/Marcotte-Bourdeau.pdf >. Consulté le 6 décembre 2009 .

McKercher (Bob) \& Du Cros (Hilary). 2002. Cultural Tourism : The Partnership between Tourism and Cultural Heritage Management. New York : New York Haworth Hospitality Press.

McKercher (Bob) \& Du Cros (Hilary). 2003. « Testing a Cultural Tourism Typology ». International Journal of Tourism Research, 5, p. $45-58$.

Ministerio de comercio exterior y turismo (Mincetur). 2011. « Capacidad Ofertada ». Site internet du Mincetur. Publication en ligne : <http://www.mincetur.gob.pe/turismo/estadistica/OfertaHotelera/Cap_Ofertada.asp>. Consulté le 2 mars 2013.

Ministerio de comercio exterior y turismo (Mincetur). 2013. " Estadísticas ». Site internet du Mincetur. Publication en ligne : $<$ http://www.mincetur.gob.pe/newweb/portals/o/turismo/sitios\%2oturisticos/Are_MONAST_STA_CATALINA_LLeg_Nac_Extr.pdf>. Consulté le 2 mars 2013.

Miranda (Carolina A.) et al. 2010. Pérou. Paris : Lonely Planet. [Septième éd.]

Morisset (Lucie K.). 1999. " Entre la ville imaginaire et la ville identitaire. De la représentation à l'espace », p. 5-36, in Ville imaginaire, ville identitaire : Échos de Québec / sous la direction de Lucie K. Morisser, Luc Noppen \& Denis Saint Jacques. Québec : Éd. Nota Bene.

Morisset (Lucie K.). 2011. « Pour une herméneutique des formes urbaines. Morphogénétique et sémiogénétique de la ville », p. 33-59, in La Ville, phénomène de représentation / sous la direction de Lucie K. Morisset \& Marie-Ëve Breton. Québec : Presses de l'université du Québec.

Morisset (Lucie K.). 2009. Des régimes d'authenticité : Essai sur la mémoire patrimoniale. Québec / Rennes : Presses de l'université du Québec / Presses universitaires de Rennes.

Organisation mondiale du tourisme (OMT). 2011. « Recommandations d'Assouan en vue d'optimiser le rôle du tourisme dans le rapprochement des cultures ». OMT. Publication en ligne : <http://media.unwto.org/fr/ content/recommandations-d-assouan-envue-d-optimiser-le-role-du-tourisme-dans-le-rapprochement-des-c>. Consulté le 2 mars 2013.

Organisation mondiale du tourisme (OMT). 2014. « Le tourisme international surpasse les attentes avec des arrivées en hausse de 52 millions en $2013 »$ » OMT. Publication en ligne : <http://media.unwto.org/fr/press-release/2014-01-20/le-tourisme-internationalsurpasse-les-attentes-avec-des-arrivees-en-hausse>. Consulté le 2 mars 2013.

Park (Hyung yu). 2010. « Heritage tourism : Emotional Journeys into Nationhood ». Annals of Tourism Research, 37, p. 116-135.

Patin (Valéry). 1988. "L'image du patrimoine et la consommation touristique », p. 19-25, in Diffusion culturelle et Exploitation touristique. Avignon : RMG Centre de congrès du palais des papes.

Patin (Valéry). 2005. Tourisme et Patrimoine. Paris : Éd. La Documentation française.

Pendlebury (John), Short (Michael) \& While (Aidan). 2009. « Urban World Heritage Sites and the Problem of Authenticity ». Cities, 26, p. 349-358.

Pertur. 2010. "Plan estratégico regional de turismo Arequipa ». Site internet de Concytec. Publication en ligne : <http://www.concytec.gob.pe/portalsinacyt/images/stories/corcytecs/arequipa/pertur_arequipa_2011-2015.pdf >. Consulté le 2 mars 2013

Poria (Yaniv), Butler (Richard) \& Airey (David). 2001. « Clarifying Heritage Tourism ». Annals of Tourism Research, 28, 4, p. 10471049.

Poria (Yaniv), Butler (Richard) \& Airey (David). 2003. « The Core of Heritage Tourism ». Annals of Tourism Research, 30, p. 238-254. Promperu. 2010. " Perfil del turista extranjero 2010 ». Site internet de Promperu. Publication en ligne : <http://intranet.promperu.gob.pe/IMPP/2010/TurismoReceptivo/Demanda\%20Actual/Perfil\%20del\%20Turista\%20Extranjero\%202010/Publicación\%20PTE\%202010.pı Consulté le 2 mars 2013.

Rachowiecki (Rob). 2000. Peru. Victoria : Lonely Planet Publications Pty Ltd. [Quatrième éd.]

Rátz (Tamara) \& Puczkó (László). 2003. « A World Heritage Industry ? Tourism at Hungarian World Heritage Sites », p. 467-482, in Regards croisés sur le patrimoine dans le monde à l'aube du XXIe siècle / sous la direction de Maria Gravari-Barbas \& Sylvie Guichard-Anguis. Paris : Presses de l’université Paris-Sorbonne.

Robinson (Mike). 2000. Tourism and Heritage Relationships : Global, National and Local Perspectives. Newcastle : Centre for Travel and Tourism/university of Northumbria.

Routard. 2013. " Arequipa et sa région ». Site internet $d u$ Guide du Routard. Publication en ligne : <http://www.routard.com/guide_voyage_lieu/3072-arequipa.htm>. Consulté le 2 mars 2013.

TripAdvisor. 2013. " Arequipa ». Site internet de TripAdvisor. Publication en ligne : <http://www.tripadvisor.fr/Tourism-g294313Arequipa_Arequipa_Region-Vacations.html>.Consulté le 2 mars 2013.

Unesco. 1972. Convention concernant la protection du patrimoine mondial, culturel et naturel, adoptée par la conférence générale dans sa 17e session, Paris, 16 novembre 1972. Paris : Unesco.

Unesco. 2013, « Centre historique de la ville d'Arequipa ». Publication en ligne : <http://whc.unesco.org/fr/list/1016/>. Consulté le 2 mars 2013

Urbain (Jean-Didier). 1998. Secrets de voyage : Menteurs, imposteurs et autres voyageurs impossibles. Paris : Payot \& Rivages.

Van der Aa (Bart J. M.). 2005. Preserving the Heritage of Humanity ? Obtaining World Heritage Status and the Impacts of Listing. Th. Doct., géographie : université de Groningue, Groningue.

\section{Notes}

2 Le ministère du Commerce extérieur et du Tourisme (Mincetur) compte le nombre de touristes qui visitent la ville d'Arequipa à partir des entrées enregistrées au monastère Santa Catalina.

3 Ce surnom de «Ville blanche » existe aussi dans de nombreuses autres villes dans le monde (Popayan, Tel Aviv). En Amérique latine cependant, il peut avoir une connotation particulière, d'abord parce qu'il rappelle la légendaire ville de Xucutaco en Amérique centrale, mais surtout parce qu'il rappelle la ville antique idéale grecque dont toutes les villes coloniales s'inspirent à travers leur plan hippodamien (orthogonal).

\section{Pour citer cet article}


Référence papier

Mathieu Dormaels, « Représentations et médiation du patrimoine mondial : Le site d'Arequipa », Culture \& Musées, 23 | 2014, 109138.

Référence électronique

Mathieu Dormaels, « Représentations et médiation du patrimoine mondial : Le site d'Arequipa », Culture \& Musées [En ligne], 23 | 2014, mis en ligne le 19 juin 2018, consulté le 20 juin 2021. URL : http://journals.openedition.org/culturemusees/1376 ; DOI : https://doi.org/10.4000/culturemusees.1376

\title{
Auteur
}

\section{Mathieu Dormaels}

Mathieu Dormaels est chercheur postdoctoral (bourse du fonds québécois de recherche, Société et Culture) à la chaire de recherche du Canada en patrimoine bâti de l'université de Montréal et professeur associé au département d'études urbaines et touristiques de l'université du Québec à Montréal. Ses recherches actuelles portent sur l'influence des représentations touristiques dans la patrimonialisation des centres-ville inscrits sur la Liste du patrimoine mondial et sur l'intégration des habitants dans les processus de patrimonialisation et de gestion des sites. Il est membre associé à la chaire de recherche du Canada en patrimoine urbain à l'université du Québec à Montréal (Canada) et à l'équipe Culture et Communication du Centre Norbert Élias (UMR 8562, France). II a récemment codirigé l'ouvrage collectif : La Patrimonialisation de l'urbain, publié aux Presses de l'université du Québec. mat_dormaels[at]yahoo.com

\section{Droits d'auteur}

\author{
Culture \& Musées
}

